\title{
Hierarchical non-negative matrix factorization (hNMF): a tissue pattern differentiation method for glioblastoma multiforme diagnosis using MRSI
}

\author{
Yuqian Li ${ }^{a, b, c *}$, Diana M. Sima ${ }^{b, c}$, Sofie Van Cauter ${ }^{d, e}$, Anca R. Croitor Sava ${ }^{b, c}$, \\ Uwe Himmelreich ${ }^{\mathrm{e}}$, Yiming $\mathrm{Pi}^{\mathrm{a}}$ and Sabine Van Huffel ${ }^{\mathrm{b}, \mathrm{c}}$
}

\begin{abstract}
MRSI has shown potential in the diagnosis and prognosis of glioblastoma multiforme (GBM) brain tumors, but its use is limited by difficult data interpretation. When the analyzed MRSI data present more than two tissue patterns, conventional non-negative matrix factorization (NMF) implementation may lead to a non-robust estimation. The aim of this article is to introduce an effective approach for the differentiation of GBM tissue patterns using MRSI data. A hierarchical non-negative matrix factorization (hNMF) method that can blindly separate the most important spectral sources in short-TE ${ }^{1}$ H MRSI data is proposed. This algorithm consists of several levels of NMF, where only two tissue patterns are computed at each level. The method is demonstrated on both simulated and in vivo short-TE ${ }^{1} \mathrm{H}$ MRSI data in patients with GBM. For the in vivo study, the accuracy of the recovered spectral sources was validated using expert knowledge. Results show that hNMF is able to accurately estimate the three tissue patterns present in the tumoral and peritumoral area of a GBM, i.e. normal, tumor and necrosis, thus providing additional useful information that can help in the diagnosis of GBM. Moreover, the hNMF results can be displayed as easily interpretable maps showing the contribution of each tissue pattern to each voxel. Copyright $\odot 2012$ John Wiley \& Sons, Ltd.
\end{abstract}

Keywords: hierarchical non-negative matrix factorization (hNMF); non-negative matrix factorization (NMF); blind source separation (BSS); MRSI; glioblastoma multiforme (GBM)

\section{INTRODUCTION}

Glioblastoma multiforme (GBM), the most common and aggressive type of brain tumor in adults, is characterized by extensive heterogeneity. Thus, the tumoral region of GBMs can consist of several tissue patterns, namely normal tissue (hereafter simply called 'normal'), actively growing tumor tissue (referred to as 'tumor') and necrotic tissue consisting of dead cells (referred to as 'necrosis') $(1,2)$. Moreover, GBMs are highly infiltrative and present patterns very similar to those of other brain tumors (i.e. metastasis, lower grade gliomas). These characteristics have posed serious difficulties in the diagnosis and prognosis of GBM. The identification and localization of normal, tumor and necrosis patterns can provide added value to the clinical investigation of GBMs for the guidance of therapy and determination of prognosis (i.e. the presence and amount of tumor and necrosis indicate the aggressiveness).

MRI is commonly used for the initial diagnosis of brain tumors, but with rather poor specificity $(3,4)$. With standard MRI methods ( $T_{1}-, T_{2}$-weighted imaging), it is currently not possible to reliably differentiate between necrosis, tumor and normal tissue in GBMs. Recently, MRSI (5), which combines the anatomical localization of MRI and the spectral information of MRS (6), has become increasingly popular as an additional noninvasive tool for the clinical diagnosis of brain tumors. MRSI provides spectral patterns of specific chemical contents in multiple voxels within a two- or three-dimensional observation grid, from which the spatial distribution of several metabolites can be estimated. The concentrations of metabolites change under disease
* Correspondence to: Y. Li, School of Electronic Engineering, University of Electronic Science and Technology of China, Xiyuan Dadao 2006, 611731, Chengdu, China. E-mail:Yuqian.Li@esat.kuleuven.be, yuqianli@uestc.edu.cn

a Y. Li, Y. Pi School of Electronic Engineering, University of Electronic Science and Technology of China, Chengdu, China

b Y. Li, D. M. Sima, A. R. Croitor Sava, S. Van Huffel

Department of Electrical Engineering, ESAT-SCD, KU Leuven, Leuven, Belgium

C Y. Li, D. M. Sima, A. R. Croitor Sava, S. Van Huffel

IBBT-KU Leuven Future Health Department, Leuven, Belgium

d S. Van Cauter

Department of Radiology, University Hospitals of Leuven, Leuven, Belgium

e. S. Van Cauter, U. Himmelreich

Biomedical MRI Unit/Molecular Small Animal Imaging Center, Department of Imaging and Pathology, KU Leuven, Leuven, Belgium

Abbreviations used: Ala, alanine; BSS, blind source separation; Cre, creatine; GBM, glioblastoma multiforme; Glu, glutamate; HALS, hierarchical alternating least squares; HLSVD-PRO, Hankel-Lanczos singular value decomposition with partial reorthogonalization; $h N M F$, hierarchical non-negative matrix factorization; Lac, lactate; Lip1, lipid at 1.3 ppm; Lip2, lipid at 0.9 ppm; Myo, myo-inositol; $N A A, N$-acetylaspartate; NMF, non-negative matrix factorization; NNLS, nonnegative least squares; $P C h$, phosphocholine; ppm, parts per million, a set of pseudo-units to describe the chemical shifts for ${ }^{1} H$ NMR spectra, i.e. the difference in hertz between a resonance frequency and that of a reference substance divided by the frequency of the magnetic field in megahertz; PRESS, point-resolved spectroscopy; SNR, signal-to-noise ratio 
conditions and thus can help in the separation between normal brain and brain tumors $(7,8)$. Moreover, previous studies have shown that we can reliably detect necrotic tissue with MRSI (9-11).

Several factors of MRSI spectral variability, such as the partial volume effect (i.e. the presence of several tissue patterns within one voxel), the chemical shift displacement effect (i.e. spatial mismatch between the excited volume of interest for different metabolites, resulting in suppressed or enhanced contributions from some metabolites in voxels along the borders of the selected volume), magnetic field inhomogeneity and measurement noise cause difficulties in the interpretation of multi-voxel MRSI data. In this context, blind source separation (BSS) techniques are gaining interest from researchers for the analysis of MRS(I) data as they have shown certain interesting properties, such as a straightforward approach for the analysis of the data without the need for prior knowledge, a better interpretation of the complexity of the data by allowing the decomposition of the mixture of acquired spectra under investigation and easily interpretable results for clinicians. Thus, under the assumption that MRSI data are linear mixtures of tissue-specific spectral profiles, called sources (or constituent spectra), BSS methods can estimate the sources and their mixing coefficients (or abundances) in a set of mixed spectra, without (or with very little) prior knowledge. For example, Ochs et al. (12) used a Bayesian framework to simultaneously obtain sources and their abundances. Szabo de Edelenyi et al. (13) detected tissue patterns inside the tumor region using independent component analysis. Non-negative matrix factorization (NMF), firstly presented by Lee and Seung (14), is a BSS method which does not require the sources to be independent or orthogonal. It fits very well with the fact that the constituent MRSI spectra of brain tumors are highly correlated in many cases. NMF was first introduced to solve the tissue pattern differentiation problem on long-TE MRSI data by Sajda et al. (15). Their constrained NMF has been proven to be a very efficient tool for the extraction of physically meaningful spectral sources (15-17). The applications in these articles focus on distinguishing between abnormal and normal brain tissue. However, for GBMs there are two distinct patterns of abnormal tissues, namely tumor and necrosis.

In this article, we propose a novel hierarchical non-negative matrix factorization (hNMF) method to accurately estimate the three most important tissue patterns for GBMs (normal, tumor and necrosis). Its benefits are proven on short-TE MRSI data. The proposed method recovers each tissue pattern step by step. An optimized thresholding procedure is employed to ensure that the three recovered spectra are as uncorrelated as possible. Spatial distributions are estimated using non-negative least squares (NNLS) fitting (18). Preliminary studies have been presented previously $(19,20)$. Here, we describe the proposed hNMF method. We then evaluate its performance in comparison with one-level NMF (direct application of NMF with the number of sources chosen to be three) and expert labeling in a simulation and an in vivo study.

\section{MATERIALS}

\section{Data acquisition protocol}

The MRSI data were acquired at the University Hospital of Leuven (UZ Leuven) on a 3-T MR scanner (Achieva, Philips, Best, the Netherlands), using a body coil for transmission and an eight-channel head coil for signal reception. The MRSI protocol had the following imaging parameters: point-resolved spectroscopy (PRESS) (21) was used as the volume selection technique; $\mathrm{TR} / \mathrm{TE}=2000 / 35 \mathrm{~ms}$; field of view, $16 \mathrm{~cm} \times 16 \mathrm{~cm}$; volume of interest, $8 \mathrm{~cm} \times 8 \mathrm{~cm}$ (maximum size); slice thickness, $1 \mathrm{~cm}$; acquisition voxel size, $1 \mathrm{~cm} \times 1 \mathrm{~cm}$; reconstruction voxel size, $0.5 \mathrm{~cm} \times 0.5 \mathrm{~cm}$; receiver bandwidth, $2000 \mathrm{~Hz}$; samples, 2048; number of signal averages, 1; water suppression method, MOIST; shimming, pencil beam shimming; first and second order, parallel imaging with SENSE factor: left-right, 2; anteriorposterior, 1.8; 10 circular 30-mm outer-volume saturation bands in order to avoid lipid contamination from the skull.

\section{Patients}

In this study, we used $12 \mathrm{MRSI}$ datasets for the purpose of validating the proposed method. The MRSI data were acquired prior to any treatment from 10 patients with brain tumors that were subsequently diagnosed as GBM. The diagnosis was based on histological examination and followed the rules of the World Health Organization (WHO) classification for tumor grading (22). These 12 datasets were visually validated as having sufficient quality $(23,24)$ and as containing three tissue patterns (normal, tumor and necrosis). The institutional review board approved the study. Written informed consent was obtained from all patients before their participation in the study.

\section{In vivo data preprocessing}

The raw MRSI data were exported from the Philips platform after standard post-processing (zero filling in $k$ space, transformation from $k$ space to normal space, automatic phase correction and eddy current correction).

The MRSI PRESS excitation volumes varied in size from patient to patient, with a maximum size of $16 \times 16$ voxels. In the MRSI PRESS excitation volume, some spectra of insufficient quality were excluded according to the criteria described in refs. $(23,24)$ [low signal-to-noise ratio (SNR), baseline distortion, large linewidths and asymmetric lineshapes as a result of poor shimming, contamination by significant chemical shift displacement effects, etc.]. The low-quality spectra were usually at the border of the PRESS excitation volume, where significant lipid contamination and obvious distortion from the chemical shift displacement effect were present. For each dataset, the percentage of discarded voxels varied according to the different number of low-quality spectra identified in each case. The final size of each selected grid (within the PRESS excitation volume) ranged from $5 \times 8$ to $12 \times 14$ (Table 1 ).

We removed the residual water components using HankelLanczos singular value decomposition with partial reorthogonalization (HLSVD-PRO) (25), setting the model order to 30 and the passband from 0.25 to 4.2 parts per million (ppm). The in-house software, SPID (26), developed on the Matlab platform, was used. The spectra were aligned in frequency using the unsuppressed water signals as reference frequency. The real parts of the preprocessed spectra were truncated to the region $0.25-4.2 \mathrm{ppm}$, resulting in $m=519$ points. Spectral normalization was not necessary as spectra of different scales for different patients did not influence the NMF results. To guarantee the non-negativity for NMF, the negative values were set to zero as they were caused by noise. 


\section{Simulation study}

We simulated an MRSI grid which mimics real data for patients with GBM. The simulated grid contained 99 voxels of spectra specific to normal tissue, nine spectra specific to tumor tissue, two spectra specific to necrosis and 33 mixed spectra (21 normal/tumor and 12 tumor/necrosis). The mixed spectra were linear combinations of spectra specific to different tissues in different percentages (see Fig. 1e). The simulated spectra were combinations of nine metabolite profiles (two simulated lipid profiles, Lip1 and Lip2, and seven metabolite profiles measured in vitro on the same scanner as the patients; see the 'Data acquisition protocol' section) and included creatine (Cre, singlets at 3.03 and $3.9 \mathrm{ppm}$ ), glutamate (Glu, between 2.04 and $2.35 \mathrm{ppm}$ and at $3.74 \mathrm{ppm}$ ), myo-inositol/ glycine-containing region (Myo, broader resonance at around $3.56 \mathrm{ppm})$, phosphocholine ( $\mathrm{PCh}$, peak at about $3.22 \mathrm{ppm})$, $\mathrm{N}$-acetylaspartate (NAA, singlet at $2.01 \mathrm{ppm}$ ), alanine (Ala, doublet at $1.47 \mathrm{ppm}$ ), lactate (Lac, doublet at $1.33 \mathrm{ppm}$ ), lipid at $1.3 \mathrm{ppm}$ (Lip1) and lipid at 0.9 ppm (Lip2). These metabolites were chosen as they are significant biomarkers for normal brain tissue, tumoral tissue and necrotic tissue in pathology (8) with the considered NMR protocol.

For each tissue pattern, we considered the spatial variability of the simulated spectra from different voxels by randomly generating the amplitude and damping (line broadening) of each metabolite around a mean value with a certain standard deviation (see Appendix A). As phase and frequency shifts can usually be reliably corrected in preprocessing procedures, these two parameters were set to zero.

The simulated time-domain MRSI signals, each having 2048 points, were Fourier transformed into the frequency domain, and the spectra obtained were then truncated from point 442 to point 960 , which corresponds to the frequency range of interest: $0.25-4.2 \mathrm{ppm}$.

In order to emulate the variability caused by measurement noise within an MRSI grid, white Gaussian noise was independently added to the time-domain signal of each voxel before Fourier transformation. The SNR was computed in the time domain as the ratio of the power of the signal to the noise standard deviation. For a time-domain signal $y$, the power of the signal is computed as the squared Euclidean norm of the time-domain signal $\|y\|^{2}$ divided by the length $m$ of the spectrum, transformed to $\mathrm{dB}$ :

$$
P_{\text {sig }}=10 \log _{10} \frac{\|y\|^{2}}{m}
$$

Examples of the simulated tissue-specific spectra are given in Fig. $1 b-d$, showing how different SNR levels influence the spectra. Examples of the in vivo spectra from a patient with GBM are also given in Fig. 1a for comparison.

\section{METHODS}

\section{Basics about NMF on MRSI data}

NMF (14) is a family of BSS algorithms, in which a matrix $X$ is approximately factorized into the product of two matrices $W$ and $H$. NMF enforces the constraint that the factors $W$ and $H$ must be non-negative. For short-TE ${ }^{1} \mathrm{H}$ MRSI spectra, the metabolic information is exhibited as peaks (either singlets or multiplets, possibly with some overlap) pointing upwards in the real part of the spectra (in absorption mode). After the preprocessing steps described above, these spectra are non-negative. Thus, tissue pattern differentiation based on MRSI spectra can be viewed as an NMF problem, as follows: all $n$ spectra from a selected region within an MRSI excitation volume can be viewed as columns of a matrix $X$, and each spectrum can be approximated by a linear mixture of unknown spectral sources pertaining to $r$ different tissue patterns (16). This gives the model:

$$
X_{m \times n}=W_{m \times r} H_{r \times n}+\text { Noise }
$$

where $m$ represents the number of points in each spectrum, $W$ is an $m \times r$ matrix and each column represents the spectral source
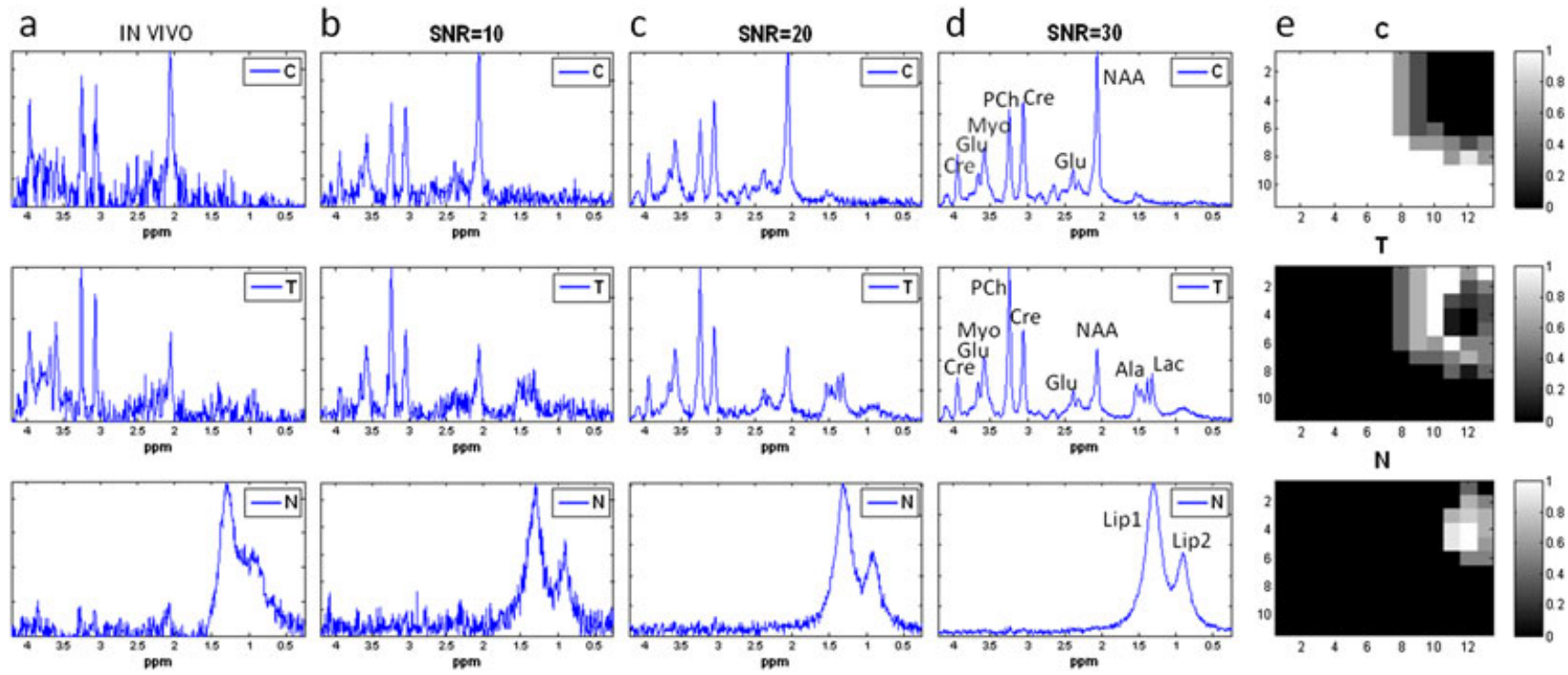

Figure 1. (a) In vivo MRSI spectra from a patient with glioblastoma multiforme (GBM). (b)-(d) Simulated MRSI spectra reflecting different tissue patterns and variable noise levels: signal-to-noise ratio (SNR) $=10$ (b), 20 (c) and 30 (d). (e) The corresponding simulated spatial distributions for each tissue type, where the scale goes from black (no contribution) to white (100\% contribution). C, T and N represent normal, tumor and necrosis, respectively. Ala, alanine; Cre, creatine; Glu, glutamate; Lac, lactate; Lip1, lipid at 1.3 ppm; Lip2, lipid at 0.9 ppm; Myo, myo-inositol; NAA, N-acetylaspartate; PCh, phosphocholine. 
for a specific tissue pattern. Each row of the $r \times n$ matrix $H$ contains the mixing coefficients (interpreted here as abundances or concentrations of each tissue pattern in each voxel, which can only take non-negative values). By reshaping each row of $H$ to the size of the selected region within the MRSI excitation volume, we can obtain the spatial distribution (hereafter referred to as 'h-map') of the corresponding constituent spectrum. 'Noise' is the additive measurement noise.

The mathematical formulation of the basic NMF problem to perform the factorization in Equation ([1]) is:

$$
\begin{gathered}
\min _{W, H} f(W, H)=\frac{1}{2}\|X-W H\|_{F}^{2} \\
\text { subject to } \forall i j, \quad W_{i j}, \quad H_{i j} \geq 0
\end{gathered}
$$

Based on the work of Lee and Seung (14), a large number of NMF algorithms have been proposed to solve the factorization problem in Equation ([2]) (27-31). The results in this article were obtained with the hierarchical alternating least squares (HALS) algorithm $(29,30)$, because HALS was proven to be superior to other NMF implementations on simulated MRSI data in our preliminary study (19).

\section{hNMF for GBM}

In the following, we present the hNMF method, which is designed to recover the three most distinct tissue-specific spectral patterns (i.e. normal, tumor and necrosis) in MRSI data from patients with GBM, as well as their corresponding spatial distributions (as shown in Fig. 2).

\section{Step 1}

First-level NMF. NMF is applied to the matrix of all spectra within the selected grid with the number of sources chosen to be two. Two spectral profiles and their corresponding h-maps $\left(H_{\text {normal }}\right.$ and $\left.H_{\text {abnormal }}\right)$ are recovered. The spectral sources obtained are automatically assigned to 'normal' and 'abnormal' tissue ( $W_{\text {normal }}$ and $W_{\text {abnormal }}$ ) based on the NAA/Lips ratio (where the NAA and Lips values are estimated as the maximum intensity in the frequency regions around 2.01 and $1.3 \mathrm{ppm}$, respectively). The source with the higher NAA/Lips ratio corresponds to normal tissue and the source with the lower NAA/Lips ratio corresponds to abnormal tissue (8).

\section{Step 2}

Second level NMF. NMF is performed with two sources repeatedly, on several sets of voxels, and the best result is chosen, as follows. Let us consider a variable threshold $t$ on the magnitude of the $H_{\text {abnormal }}$ map, according to which a mask $f(t)$ is created, i.e. a selection of 'abnormal' voxels having values of $H_{\text {abnormal }}$ larger than $t$. For any fixed value of $t$, NMF with the number of sources chosen to be two is applied to the set of currently selected 'abnormal' spectra, leading to two sources, $W_{\text {abnormal }}^{1}(t)$ and $W_{\text {abnormal }}^{2}(t)$. In order to choose the best mask $f(\hat{t})$ from a set of gradually decreasing masks, a reasonable trade-off is sought, such that $W_{\text {normal, }} W_{\text {abnormal }}^{1}(\hat{t})$ and $W_{\text {abnormal }}^{2}(\hat{t})$ are mutually least correlated, i.e.:

$$
\hat{t}=\arg \min (\operatorname{corr} 1(t)+\operatorname{corr} 2(t)+\operatorname{corr} 3(t))
$$

where:

$$
\begin{aligned}
& \operatorname{corr} 1(t)=\operatorname{corr}\left(W_{\text {normal }}, W_{\text {abnormal }}^{1}(t)\right) \\
& \operatorname{corr} 2(t)=\operatorname{corr}\left(W_{\text {normal }}, W_{\text {abnormal }}^{2}(t)\right) \\
& \operatorname{corr} 3(t)=\operatorname{corr}\left(W_{\text {abnormal }}^{1}(t), W_{\text {abnormal }}^{2}(t)\right)
\end{aligned}
$$

and $\operatorname{corr}(x, y)=\frac{(x-\bar{x})^{\top}(y-\bar{y})}{\|x-\bar{x}\| \cdot\|y-\bar{y}\|}$ for any two vectors $x$ and $y$ of the same length. $\bar{x}$ and $\bar{y}$ are the means of $x$ and $y$, respectively.

The profiles $W_{\text {abnormal }}^{1}(\hat{t})$ and $W_{\text {abnormal }}^{2}(\hat{t})$ are automatically assigned to necrosis and tumor tissue ( $W_{\text {necrosis }}$ and $\left.W_{\text {tumor }}\right)$ based on the Cho/Lips ratio (where the Cho and Lips values are estimated as the maximum intensity in the frequency regions around 3.22 and $1.3 \mathrm{ppm}$, respectively). Based on previous studies (8), the source with the smaller Cho/Lips ratio is assigned to necrosis and the other is assigned to tumor.

Step 3

NNLS re-estimation. We apply NNLS to the grid considered in step 1 using the sources $W_{\text {normal, }}, W_{\text {tumor }}$ and $W_{\text {necrosis }}$ to re-estimate the corresponding h-maps.

\section{Remarks}

(1) The $f(\hat{t})$ region selected in step 2 excludes voxels containing a mixture of normal tissue and tumor, as their presence hinders the accuracy of the second-level NMF. With step 3, we aim to re-estimate the mixture between normal and tumor tissue.

(2) At each decomposition level, the h-maps are normalized to take values between zero and unity, which means that the threshold $t$ is also bounded between zero and unity. The normalization is based on the maximum and minimum values found in one of the tissue components. If $h_{i}$ is defined as an arbitrary element of the h-map $H$, each element $h_{i}$ in $H$ is normalized in this way: normalized $h_{i}=\frac{h_{i}-\min (H)}{\max (H)-\min (H)}$

(3) For the initialization values needed by the NMF algorithm, we used simulated spectra that reflect spectral tissue patterns specific to normal, tumor and necrosis, as described in detail in ref. (19) and in the 'Simulation study' section. The h-maps are initialized with random numbers.

\section{Performance evaluation for the simulation study}

The simulation study aims to evaluate the performance of the proposed hNMF algorithm compared with the one-level NMF, i.e. the direct application of NMF with the number of sources chosen to be three. The performance was evaluated at different noise levels (SNR $=10,15,20,25,30)$. For each noise level, both algorithms were applied on the simulated MRSI data (see 'Simulation study' section).

The performance evaluation was conducted using two measures. As the first, we computed the correlation coefficient $R$ between each source estimated by hNMF and the corresponding representative tissue-specific spectrum $W^{\text {rep }}$, computed as the average of all the simulated noiseless spectra pertaining to the respective tissue pattern; for example, $R=\operatorname{corr}\left(W_{\text {normal }}, W_{\text {normal }}^{\text {rep }}\right)$ would be the correlation coefficient for the normal tissue pattern. $R$ takes values between ' -1 ' and ' 1 ', where a value close to ' -1 ' indicates a negative correlation, one close to zero that the sources are uncorrelated and one close to ' 1 ' that the sources are highly correlated with the representative tissue-specific spectra, and therefore the method reaches best performance. Then, the correlation coefficients for the three tissue patterns are summed, leading 


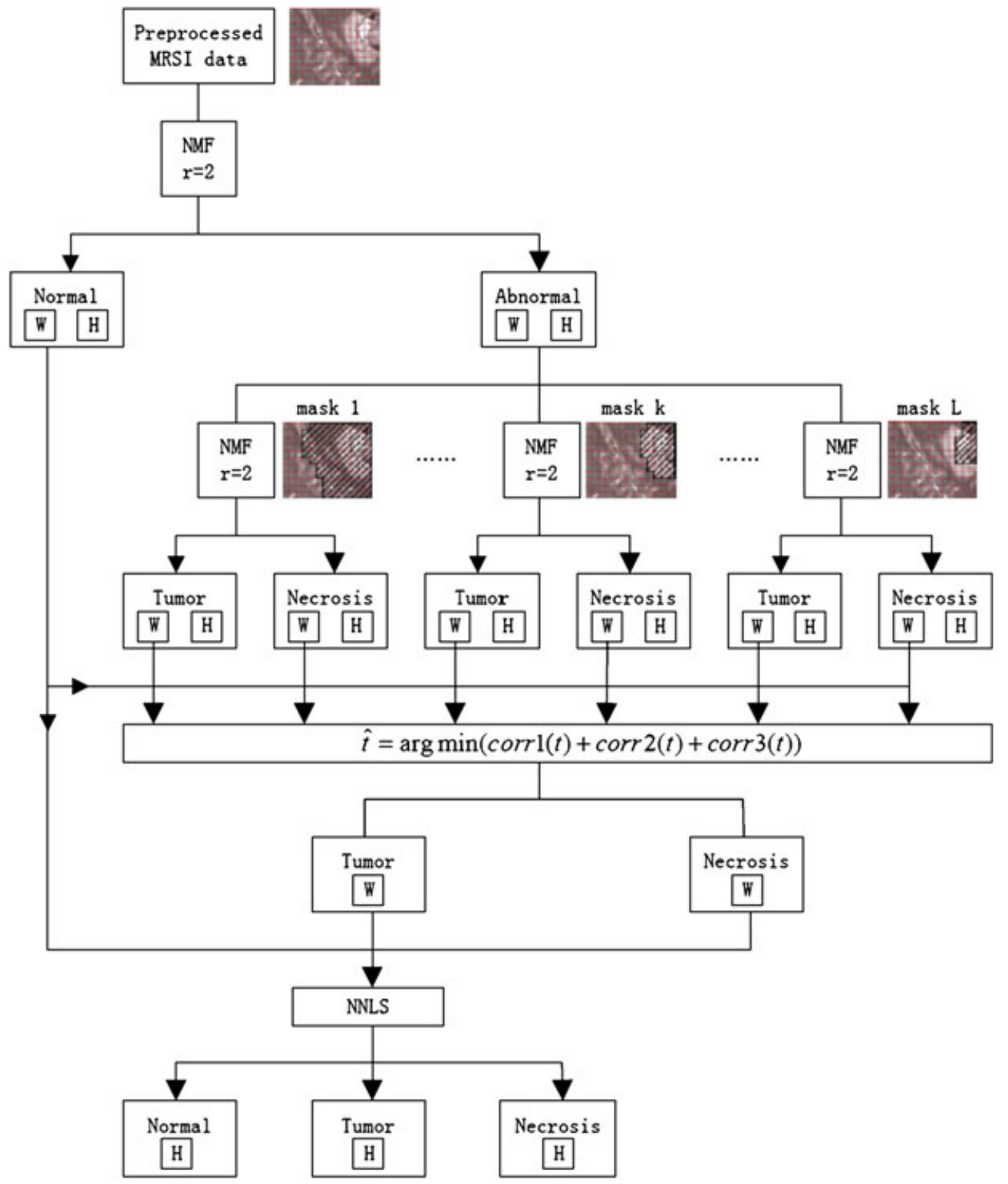

Figure 2. Scheme of the hierarchical non-negative matrix factorization (hNMF) algorithm. NNLS, non-negative least squares.

to the 'total correlation coefficient' (total $R$ ), which indicates the overall accuracy of the three recovered sources. The total $R$ is between ' -3 ' and ' 3 ', with ' 3 ' being a perfect result.

The second performance measure is the error rate of the corresponding spatial distribution (h-map) for each tissue pattern, which is calculated as:

$$
\text { Error Rate }=\frac{\sum\left(h_{\mathrm{e}}-h_{\mathrm{s}}\right)^{2}}{\sum h_{\mathrm{s}}{ }^{2}}
$$

where $h_{\mathrm{e}}$ is the estimated h-map from hNMF for that tissue pattern, $h_{\mathrm{s}}$ is the original simulated spatial distribution for the same tissue pattern and $\sum$ sums all the values in the grid. The error rates of the three tissue patterns are summed, leading to the 'total error rate', which indicates the overall accuracy of the estimated tissue distribution. Obviously, the total error rate is greater than zero, but it does not have a predefined upper bound.

\section{Performance evaluation for in vivo data}

The results of our in vivo study were also evaluated in two ways. One method involved the calculation of the total correlation coefficient (total $R$ ), in a similar manner to that described above. However, instead of comparing the hNMF sources with the simulated representative tissue-specific spectra, we compared them with 'ideal' patient-specific and tissue-specific spectra based on expert labeling of the in vivo spectra. More specifically, the 'ideal' spectrum for each tissue pattern and for each grid was computed as the average of all the spectra labeled by two experts (SVC, UH) as a certain tissue pattern, i.e., normal, tumor or necrosis, in the considered grid. Only spectra for which a consensus was reached were considered, where the decision was based on conventional $T_{1}$ - and $T_{2}$-weighted MRI pre- and post-contrast agent administration, as well as a prior knowledge of MRS patterns in tumors $(6,8)$ (e.g. increased Cho/Cre ratio and absence of NAA for tumor, lipids for necrosis). 
The second method for the validation of the in vivo results was based on a visual comparison of the h-maps with expert labeling. Numerically, it is difficult to compare the accuracy of our h-maps with expert labeling, as the h-maps contain numbers between zero and unity, whereas the experts labeled each voxel as one tissue or a mixture, without specifying percentages. As such, we examined the quality of the h-maps by overlaying them with the MR images and by comparison with expert labeling, after translating the expert labeling into color maps.

\section{RESULTS}

First, the performance of hNMF was demonstrated using simulated MRSI data from patients with GBM by comparing the results with the one-level NMF. We then applied hNMF to in vivo ${ }^{1} \mathrm{H}$ MRSI data from patients with GBM and compared the performance of the proposed algorithm with one-level NMF and expert labeling.

\section{Performance evaluation in the simulation study}

The hNMF performance, namely the total $R$ and total error rate on simulated MRSI data, is illustrated in Fig. 3 as boxplots. When increasing the SNR level, we can see that the total $R$ of hNMF increases and the total error rate of hNMF decreases. For all the noise levels, we observe that the total $R$ of hNMF is larger than the total $R$ of one-level NMF, and the total error rate of hNMF is smaller than the total error rate of one-level NMF, which indicates that hNMF performs better than one-level hNMF in recovering the underlying spectral sources from mixed MRSI spectra. We can also observe that the boxes of one-level NMF are wider than those of hNMF, thus indicating a lack of robustness of the one-level approach.

\section{Need for threshold optimization}

One of the novelties of the proposed hNMF method is the optimized thresholding procedure (step 2 in 'hNMF for GBM' section), which contributes towards finding the most distinct sources. The sources estimated at the second-level NMF can be inaccurate if the best mask is not properly chosen. To illustrate the need for threshold optimization, as given in Equations ([3]) and ([4]), an example from in vivo MRSI data is shown in Fig. 4, where the selected abnormal region under the best mask $f(\hat{t})$ is given in Fig. $4 f$ and the corresponding hNMF results in Fig. $4 \mathrm{~g}$. As can be seen, the recovered spectral profiles correspond very well to the 'ideal' profiles. Comparing the h-maps, we also notice that the h-maps in Fig. $4 \mathrm{~g}$ correspond well to expert labeling, as shown in Fig. 4c. However, if the mask is not properly chosen, the results will be partially incorrect. From Fig. 4e, the recovered tumor spectrum using the wider selected abnormal region shown in Fig. $4 d$ is very different from the 'ideal' tumor spectrum. For the selected abnormal region shown in Fig. 4h, which is much smaller than in Fig. 4f, the recovered spectra are also inaccurate. The lipids in the recovered tumor spectrum are much higher than those of the 'ideal' spectrum (note the highest peak in the middle spectrum in column Fig. 4i). For further illustration, we calculate the total $R$ between the recovered spectra and the 'ideal' spectra under these three different thresholds. For the wide mask in Fig. $4 \mathrm{~d}$, total $R=2.77$. For the small mask in Fig. $4 \mathrm{~h}$, total $R=2.69$. For the mask in Fig. $4 \mathrm{f}$, under the optimal threshold, total $R=2.94$, which is the best of the three. Therefore, such results demonstrate the importance of choosing the best threshold.

\section{Need for spatial re-estimation}

Figure 5 illustrates the need to apply NNLS (step 3 in 'hNMF for $\mathrm{GBM}^{\prime}$ section). Figure 5a shows the total error rates of the spatial distributions in the simulation study for different noise levels. As can be seen, the error rate for all noise levels before the use of NNLS is much poorer than after NNLS re-estimation. This is because the second-level NMF is applied on a mask. However, the area around the thresholded region might also contain mixed tissue. NNLS re-estimation can avoid the loss of border information between normal and tumor tissues, thus obtaining a more accurate spatial distribution for each tissue pattern over the whole grid. An example of the hNMF results based on in vivo MRSI data is also demonstrated in Fig. $5 c$. Figure $5 b$ shows the anatomical image. As can be seen, the tumor region appears to be too tightly cropped before NNLS re-estimation, whereas there are more voxels with a suspicion of some contribution from tumor tissue after re-estimation using NNLS. a

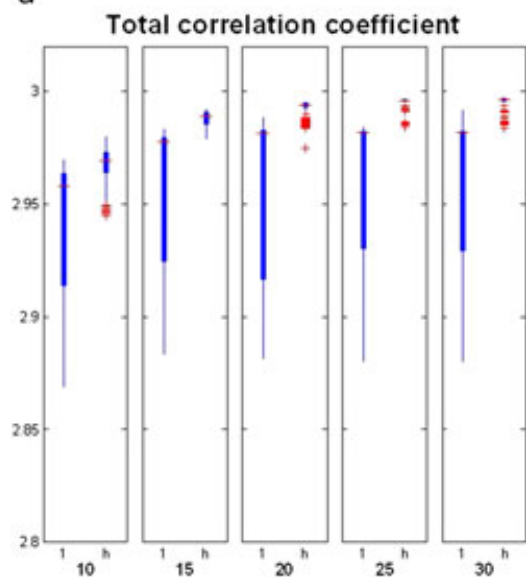

b

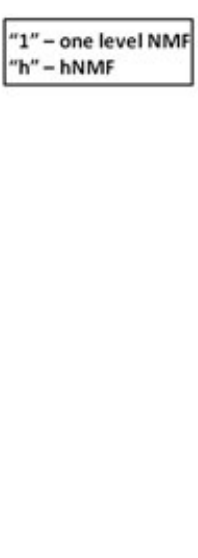

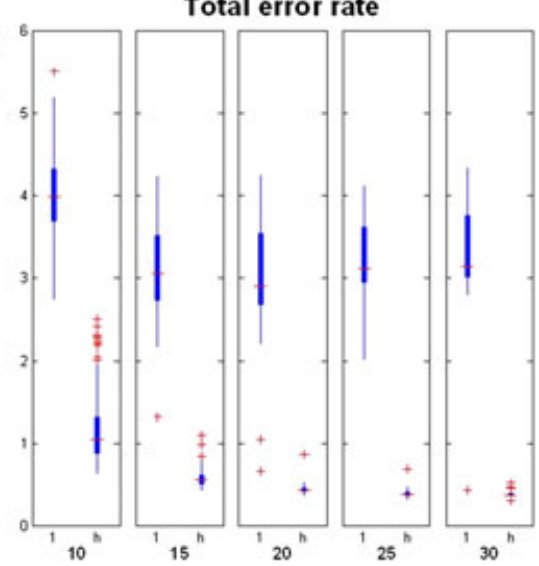

Figure 3. Performance evaluation on simulated MRSI data [signal-to-noise ratio $(S N R)=10,15,20,25,30]$. For each noise level, the left boxplot shows the results of one-level non-negative matrix factorization (NMF) with the number of sources chosen to be three, labeled as ' 1 '. The right boxplot shows the results of hierarchical non-negative matrix factorization (hNMF), labeled as ' $\mathrm{h}$ '. (a) Total correlation coefficient (total $R$ ) of the three recovered spectra. (b) Total error rate of the three estimated spatial distributions. 

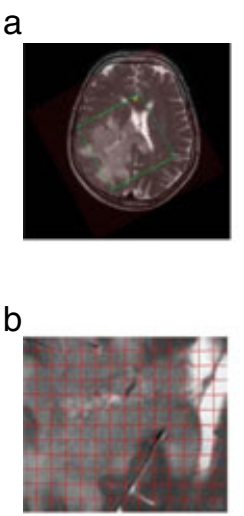

C

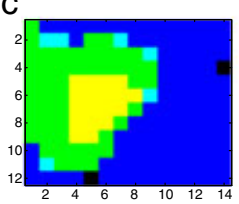

d

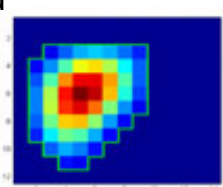

e
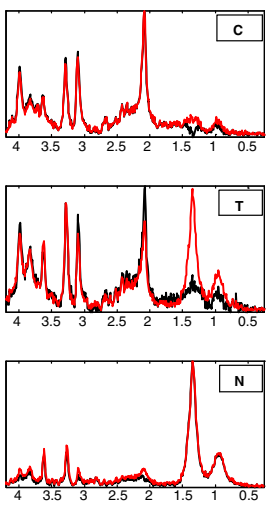

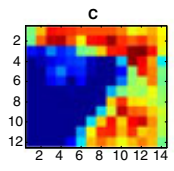

g
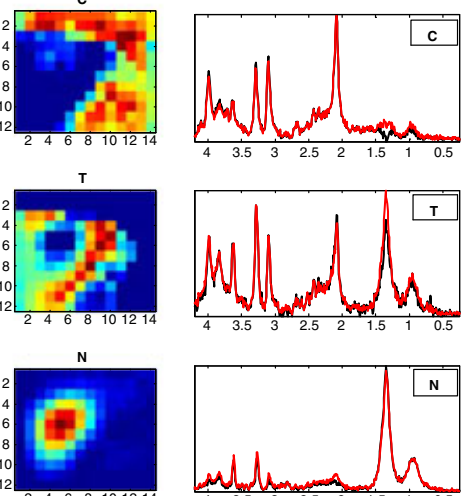

f
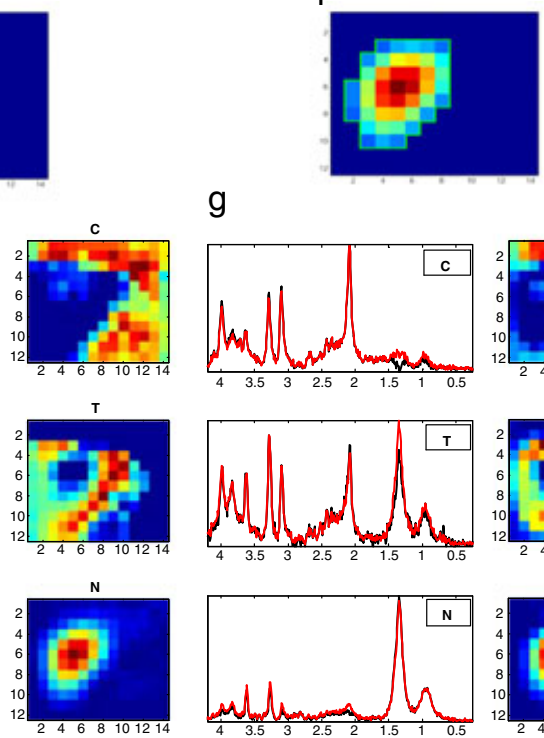

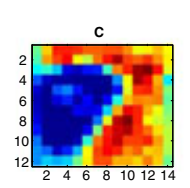

i
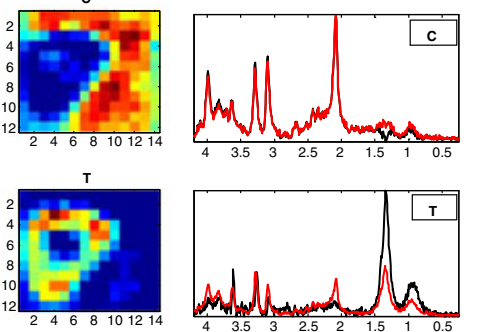

N
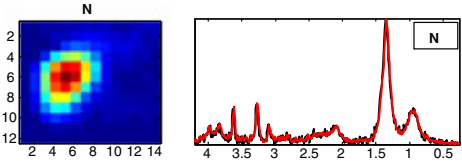

h

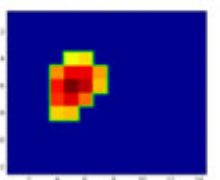

Figure 4. Illustration of the need for threshold optimization: dataset 2. C, T and N denote normal, tumor and necrosis, respectively. (a) Anatomical $T_{2}$-weighted MR image; (b) zoom on the selected grid; (c) additional information from expert labeling, where yellow indicates $\mathrm{N} / \mathrm{T}$, green indicates $\mathrm{T}$, cyan indicates $C / T$, blue indicates $C$ and black indicates spectra of poor quality; $(d, f, h)$ selected mask corresponding to three different thresholds; under each of the three masks, the results of hierarchical non-negative matrix factorization (hNMF) are shown in (e), (g) and (i), respectively, in the form of the recovered spectra and estimated h-maps for each tissue pattern. The recovered spectra using hNMF are shown in black. The red lines are the 'ideal' spectra for this patient according to expert labeling.

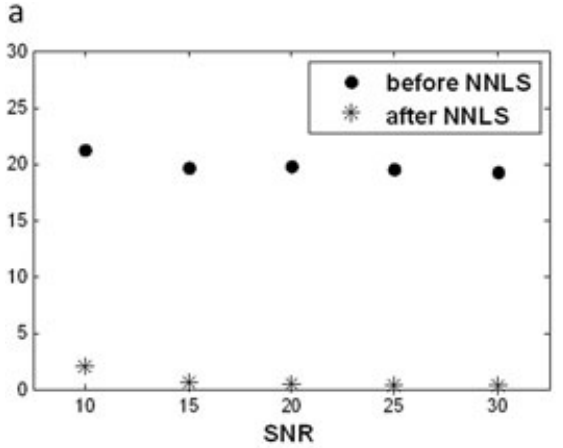

b

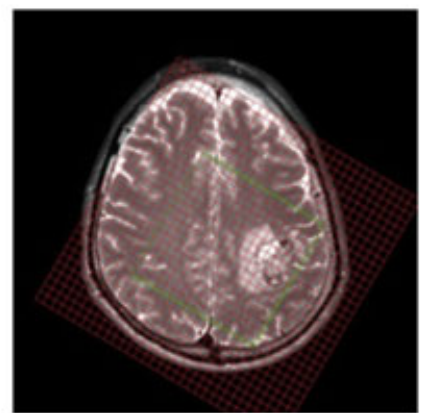

C

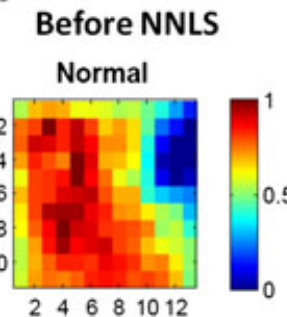

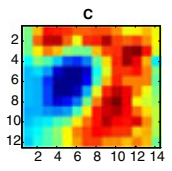

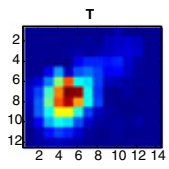

N

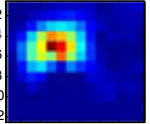

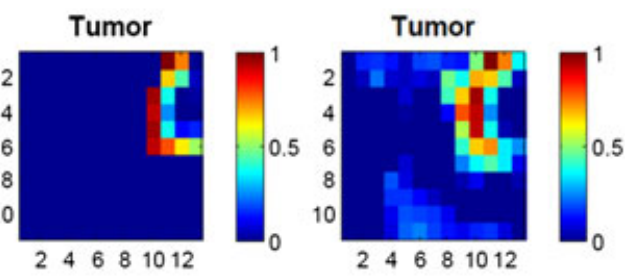

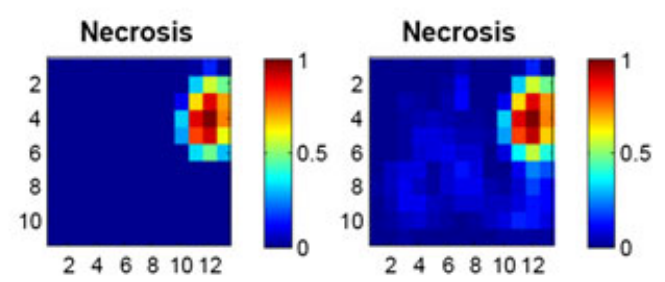

Figure 5. (a) Total error rates of spatial distributions evaluated for different noise levels on simulated MRSI data. The dots represent the total error rates calculated before the use of non-negative least squares (NNLS) and the asterisks represent the total error rates after the use of NNLS. (b) Anatomical $T_{2}$-weighted MR image: dataset 1. (c) Estimated spatial distribution of three tissue patterns on in vivo MRSI dataset 1. The left maps show the result without NNLS. The maps in the right column show the spatial distribution over the whole grid after NNLS re-estimation. SNR, signal-to-noise ratio.

\section{Validation of tissue pattern differentiation on in vivo data}

In order to validate the tissue differentiation ability for GBMs, hNMF was applied to in vivo ${ }^{1} \mathrm{H}$ MRSI data from patients with
GBM and compared with one-level NMF. As shown in Table 1, hNMF is capable of recovering the three expected tissue patterns for all the 12 datasets of patients with GBM, yielding total $R$ values between 2.70 and 2.96. One-level NMF only works in five 
Table 1. First 3 columns: Summary of in vivo MRSI datasets. Last 2 columns: Tissue differentiation comparison between hierarchical non-negative matrix factorization (hNMF) and one-level non-negative matrix factorization (NMF) for all 12 datasets that contain three tissue patterns. ' $X$ ' denotes failure to recover three sources, i.e. at least two sources have very similar patterns. For cases in which three sources were recovered, we give the total $R$ values in the table. More detailed results for dataset 1 and dataset 2 are shown in Figs 6 and 7, respectively. The entire field of view is $32 \times 32$ voxels

\begin{tabular}{|c|c|c|c|c|}
\hline Dataset & $\begin{array}{l}\text { Size (in voxels) of PRESS } \\
\text { excitation volume }\end{array}$ & $\begin{array}{l}\text { Size (in voxels) of } \\
\text { selected grid }\end{array}$ & Total $R$ of hNMF & Total $R$ of one-level NMF \\
\hline 1 & $16 \times 16$ & $11 \times 13$ & 2.94 & $x$ \\
\hline 2 & $16 \times 16$ & $12 \times 14$ & 2.96 & $x$ \\
\hline 3 & $16 \times 16$ & $7 \times 7$ & 2.74 & 2.66 \\
\hline 4 & $16 \times 16$ & $7 \times 7$ & 2.81 & $x$ \\
\hline 5 & $16 \times 16$ & $10 \times 14$ & 2.91 & 2.76 \\
\hline 6 & $16 \times 16$ & $11 \times 11$ & 2.95 & $x$ \\
\hline 7 & $16 \times 12$ & $11 \times 7$ & 2.70 & $x$ \\
\hline 8 & $16 \times 16$ & $6 \times 8$ & 2.81 & $x$ \\
\hline 9 & $16 \times 16$ & $5 \times 8$ & 2.86 & 2.62 \\
\hline 10 & $8 \times 9$ & $8 \times 7$ & 2.93 & $x$ \\
\hline 11 & $16 \times 8$ & $11 \times 7$ & 2.93 & 2.52 \\
\hline 12 & $16 \times 12$ & $11 \times 3$ & 2.90 & 2.67 \\
\hline
\end{tabular}

cases as, in the others, at least two of the three sources have very similar patterns. For those five cases in which one-level NMF works, the total $R$ of one-level NMF is smaller than that of hNMF. These numbers fully demonstrate that one-level NMF is limited in accurately revealing the underlying tissue patterns for in vivo ${ }^{1} \mathrm{H}$ MRSI data, and that hNMF is a more efficient alternative for tissue pattern differentiation.

We show two examples of in vivo experiments in Figs 6 and 7. Similar results were obtained for the other in vivo experiments. The results of hNMF are shown in Fig. $6 \mathrm{~d}$, e and Fig. $7 d$, e.

In Fig. 6a, the anatomical image in the first row clearly demonstrates a tumoral lesion with central necrosis in the left parietal lobe. The zoom on the selected grid is shown in the second row and expert labeling is translated into a color map in the third row of Fig. 6a. From Fig. 6b, c, it can be seen that the one-level NMF algorithm fails. In Fig. 6b, the recovered sources from one-level NMF are in black, overlaid with the 'ideal' spectra in red. We can see that the source showing necrosis (third row in Fig. $6 \mathrm{~b}$ ) is correctly determined by one-level NMF and corresponds to the areas of necrosis on the anatomical image. However, the first and second rows in Fig. $6 \mathrm{~b}$ both show spectral patterns of normal tissue. In this case, the normal area is separated into two subareas, as shown in Fig. 6c. The spectral differences between these two profiles are caused by the chemical shift displacement effect rather than by a biological difference in tissue patterns. By contrast, Fig. $6 \mathrm{~d}$, e shows the results of hNMF. The three spectra in black in Fig. $6 \mathrm{~d}$ represent the recovered normal, tumor and necrosis sources. They show high similarity with the 'ideal' spectra in red. The corresponding h-maps are shown in Fig. 6e and correspond very well with the anatomical image and the expert labeling. In the second row in Fig. 6e, the red color shows the higher concentration of the corresponding tumoral source shown in the second row in Fig. $6 \mathrm{~d}$. In the second row of Fig. 6e, orange, yellow, green and cyan reflect partial tumor cell content, i.e. voxels with a suspicion of tumor infiltration. The total $R$ of hNMF is 2.94, which shows a very high correlation between the recovered sources and the 'ideal' spectra. However, the total $R$ of one-level NMF is only
1.95 , which is much lower, mainly because of differences in the tumor spectral profile.

Figure 7 shows a typical case of a patient with GBM with only discrete macroscopic necrosis. One-level NMF and hNMF were applied to the selected grid as shown in the second row in Fig. 7a. The anatomical image is in the first row of Fig. 7a. Expert labeling was translated into a color map, shown in the third row in Fig. 7a. Figure $7 b, c$ shows that one-level NMF failed to recover the three correct tissue patterns. As in the first example, the differences in spectral separation are caused by the chemical shift displacement effect as the one-level NMF sources in the first two rows in Fig. 7b both represent normal tissue. The first two h-maps in Fig. 7c both represent the spatial distribution of normal tissue. The sources correctly recovered by hNMF are shown in Fig. 7d for normal, tumor and necrosis, and Fig. 7e shows the corresponding spatial distribution. Although the anatomical image in Fig. 7a does not show necrosis, the locations of these three tissue patterns were confirmed by expert labeling. The colors between red and blue in the second row in Fig. 7e reflect mixed tissue and suggest how tumor possibly infiltrates into the surrounding regions. We also calculated the total $R$ for a quantitative comparison of accuracy. The total $R$ of one-level NMF is 2.35 and that of hNMF is 2.96 , showing that the three tissue patterns are well separated in the data. These results demonstrate the efficiency and superiority of hNMF in recovering underlying sources from MRSI data.

\section{DISCUSSION AND CONCLUSIONS}

This study explored the efficiency of a new method, hNMF, for the recovery of normal, tumor and necrosis tissue patterns for patients with GBM using short-TE MRSI data. The development of this method was motivated by the fact that one-level NMF consistently fails in this task. The reason for this failure probably lies in the fact that the tissue-specific spectral profile of tumor is not sufficiently uncorrelated from a linear combination of other tissue patterns: normal and necrosis. hNMF looks for the three 


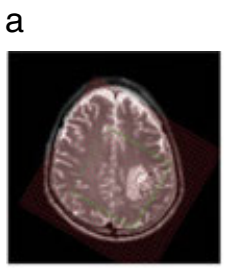

b
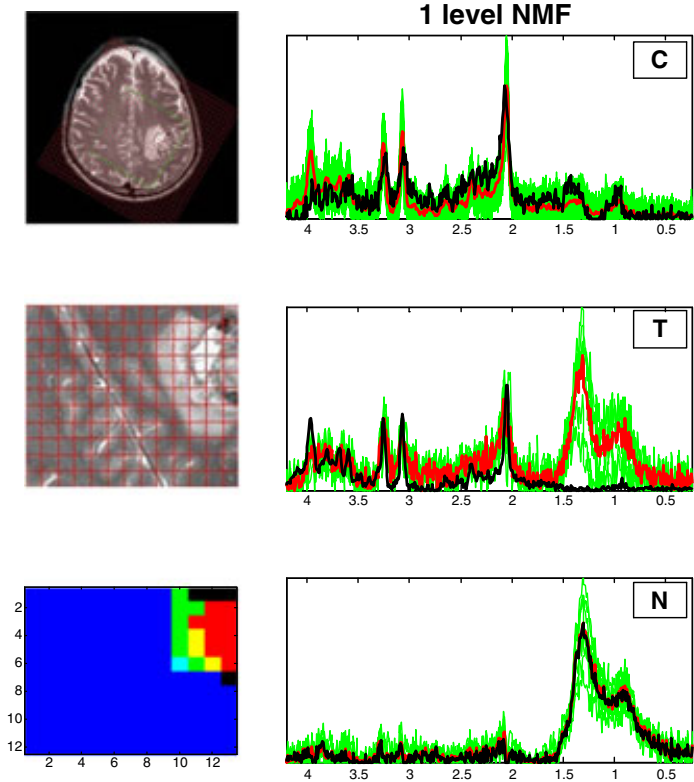
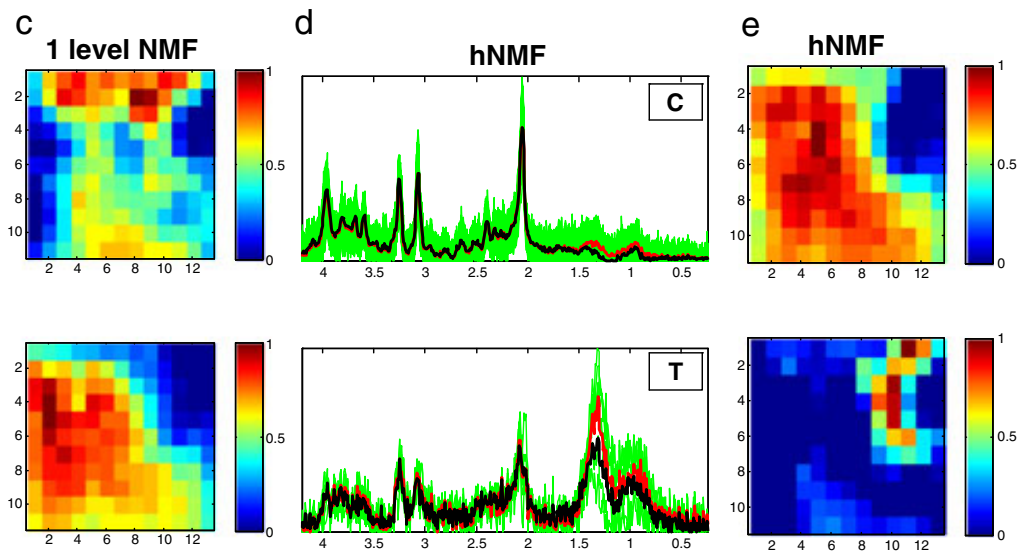

d

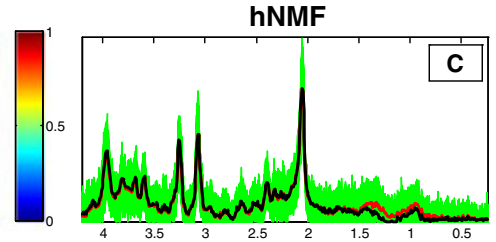

e hNMF
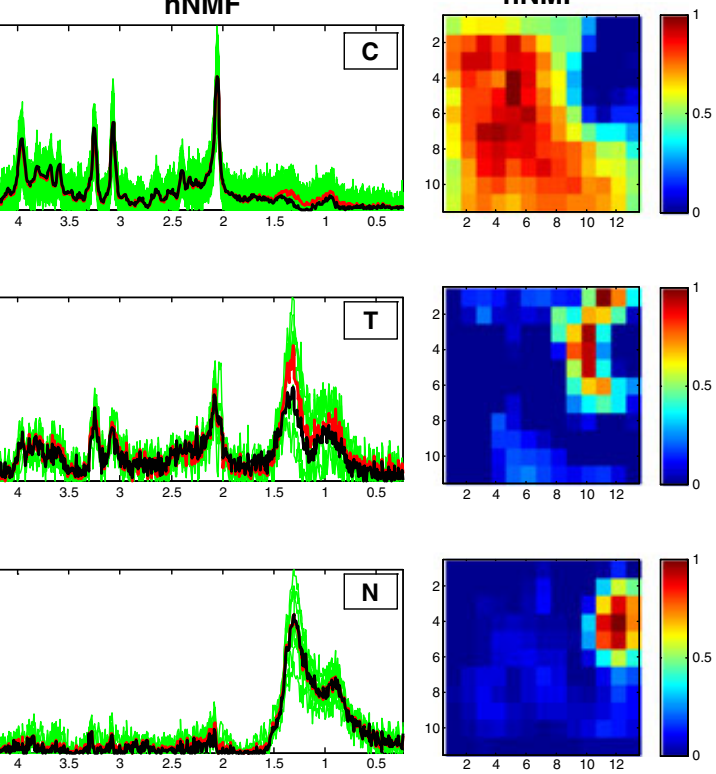

Figure 6. Tissue pattern differentiations on ${ }^{1} \mathrm{H}$ MRSI data from a patient with glioblastoma multiforme (GBM): dataset 1. C, T and $\mathrm{N}$ represent normal, tumor and necrosis, respectively. (a) First row: anatomical $T_{2}$-weighted MR image. Clear tumor and necrosis are visible in the image. Second row: selected voxels of interest. Third row: expert labeling, where red indicates $N$, yellow indicates $N / T$, green indicates $T$, cyan indicates $C / T$, blue indicates $\mathrm{C}$ and black indicates spectra of poor quality. ( $\mathrm{b}, \mathrm{c}$ ) Results of one-level non-negative matrix factorization (NMF). (b) The recovered sources are shown in black. Spectra in red are the 'ideal' spectra according to expert labeling. Spectra in green show all the tissue-specific spectra labeled by the experts. (c) Corresponding h-maps for each spectral profile given in (b). (d, e) Results of hierarchical non-negative matrix factorization (hNMF). (d) The recovered sources for C, T and $\mathrm{N}$ are shown in black, overlaid over the 'ideal' spectra in red. Spectra in green show all the tissue-specific spectra labeled by the experts. (e) Corresponding h-maps which can be compared with the anatomical image and expert labeling.

a

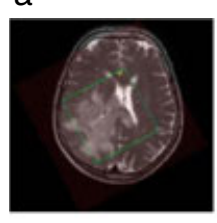

b
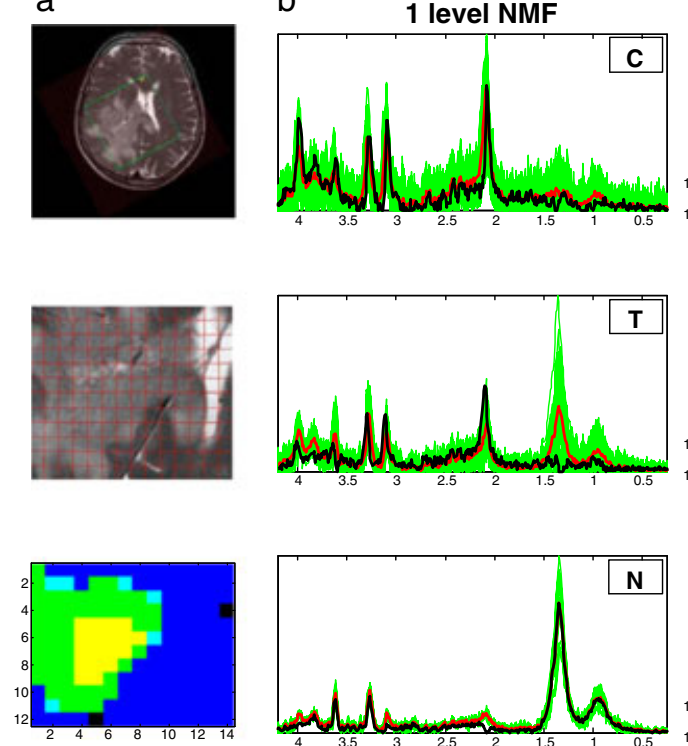
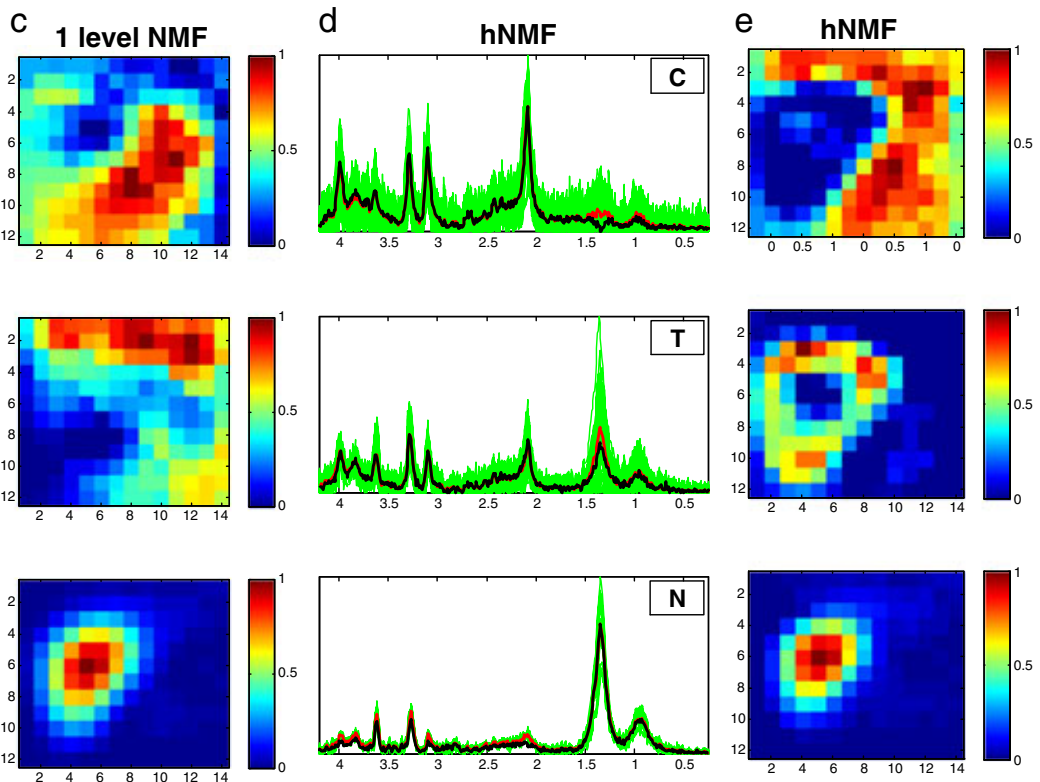

Figure 7. Tissue pattern differentiation on ${ }^{1} \mathrm{H}$ MRSI data of a patient with glioblastoma multiforme (GBM): dataset 2. C, T and N represent normal, tumor and necrosis, respectively. (a) First row: anatomical $T_{2}$-weighted MR image shows a glioblastoma with some areas of necrosis, infiltrating in the splenium of the corpus callosum. Second row: selected voxels of interest. Third row: expert labeling, where yellow indicates $N / T$, green indicates $\mathrm{T}$, cyan indicates $\mathrm{C} / \mathrm{T}$, blue indicates $\mathrm{C}$ and black indicates spectra of poor quality. (b, c) Results of one-level non-negative matrix factorization (NMF). (b) The recovered sources are shown in black. Spectra in red are the 'ideal' spectra according to expert labeling. Spectra in green show all the tissue-specific spectra labeled by the experts. (c) Corresponding h-maps for each spectral profile given in (b). (d, e) Results of hierarchical non-negative matrix factorization (hNMF). (d) The recovered sources for C, T and N are shown in black, overlaid over the 'ideal' spectra in red. Spectra in green show all the tissue-specific spectra labeled by the experts. (e) Corresponding h-maps which can be compared with the anatomical image and expert labeling. 
most distinct (but possibly correlated) tissue-specific spectral profiles by optimizing a mask on the abnormal region, which is meant to exclude voxels containing a mixture of tumor and normal tissue patterns. The mixing coefficients are finally computed as the h-maps corresponding to each tissue pattern.

A hierarchical application of NMF has also been presented in refs. $(15,17)$, but only a fixed threshold was used to generate a mask for data selection. The MRSI data used in refs. $(15,17)$ had a different protocol from ours [i.e. a multislice MRSI sequence (32) instead of PRESS localization]; consequently, the stages of the hierarchical approach were different: an initial stage was devoted to finding the brain tissue within the whole head image, and subsequent stages were meant to differentiate between normal brain tissue, tumor, water and lipids. Our hierarchical method was applied directly to brain tissue data and was able to differentiate three tissue patterns instead of only two (i.e. normal and abnormal) in the region encompassing the tumor. Another difference from refs. $(15,17)$ was the NNLS re-estimation added as the last step of the hNMF method; this avoids the loss of information at the border between tissue patterns, which may otherwise occur as a result of thresholding. In our in vivo results, it can be seen that there is no clear border between tissue patterns for GBM data. This feature can be attributed to the effect of the point spread function, but also to the infiltrative nature of glial cells.

The previous studies (15-17) were limited to long-TE ${ }^{1} \mathrm{H}$ MRSI ( $T E / T R=280 / 2300 \mathrm{~ms}$ ). However, short-TE spectra provide more diagnostic information than long-TE spectra, but are potentially more challenging than long-TE spectra because of the more complex spectral profiles. According to Majós et al. (33), more resonances are visible at short TE than at long TE because the signal intensity from compounds with strong $J$ modulation may be lost at long TE. One of their most important conclusions is that short-TE ${ }^{1} \mathrm{H}$ MRS produces a slightly more accurate diagnostic outcome in general, including the diagnosis of GBM. To date, there has been no evidence to show that tissue differentiation using NMF on short-TE spectra gives good results. Our experiments demonstrate that hNMF works properly on short-TE ${ }^{1} \mathrm{H}$ MRSI spectra.

Su et al. (16) chose to use absolute spectra instead of real-valued absorption mode spectra to guarantee non-negative spectra for the NMF algorithm, because the linearity of the model can be approximately maintained when peaks do not overlap significantly and the noise level is low. However, these two assumptions are not met in our study which uses short-TE data, where highly overlapping resonances and high noise occur. Therefore, we opted to use the real part of the spectra, similarly to the study of Szabo de Edelenyi et al. (13).

We applied the hNMF method to spectra from a single MRSI dataset at a time. Other authors have applied NMF or other BSS methods on collections of MR spectra acquired from different patients [e.g. single-voxel MRS $(34,35)]$. The advantage of working patient-wise is twofold: first, there is no need to collect a database of spectra from different patients; and, second, interindividual differences are accounted for within the method
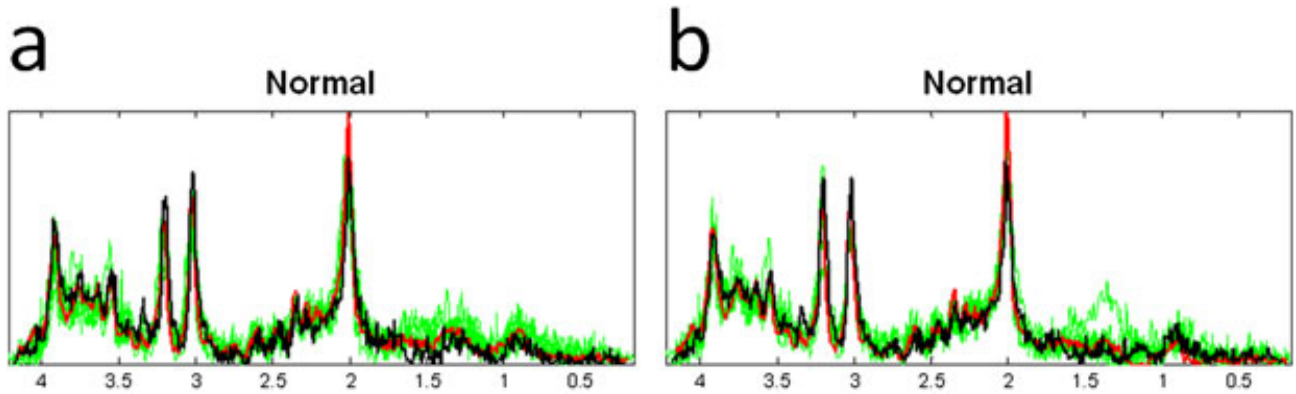

Tumor

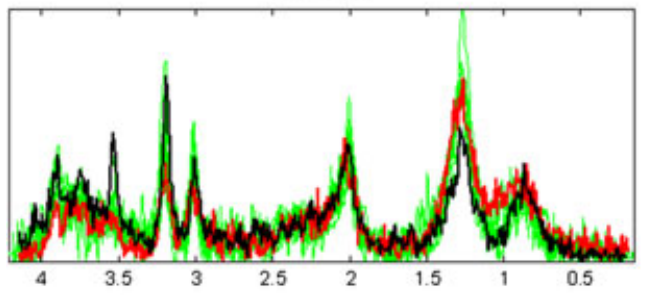

Tumor
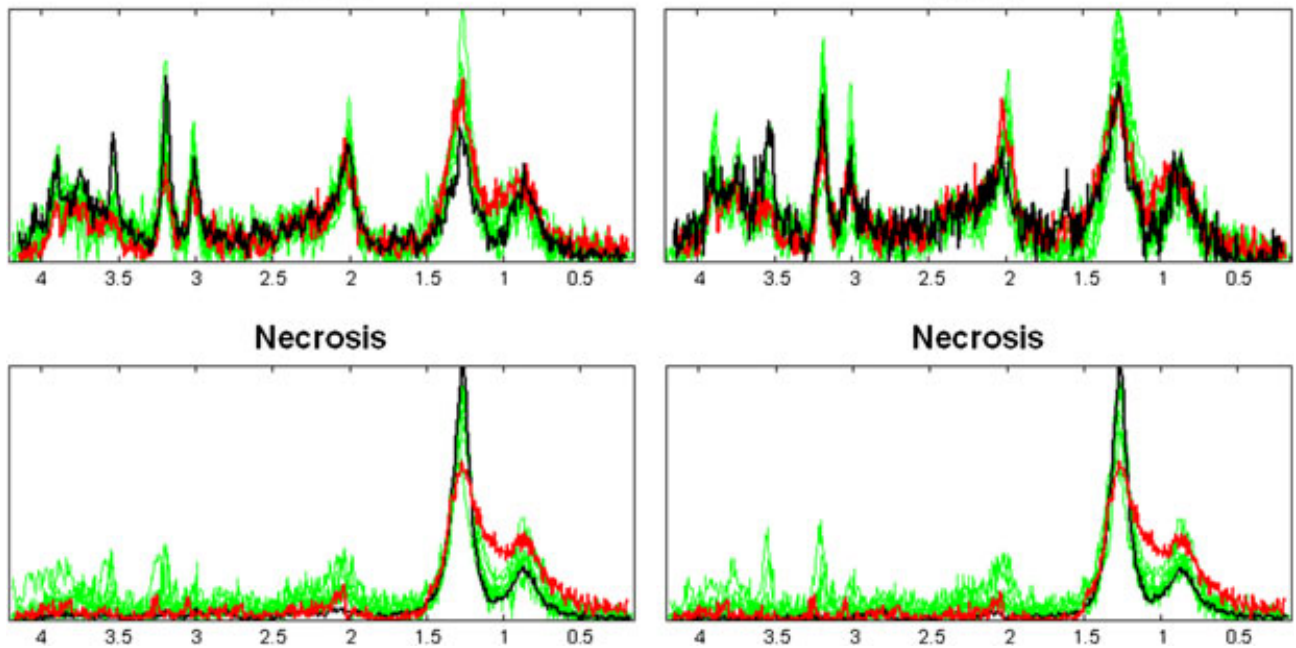

Figure 8. Variation of spectra between patients. (a) The 'ideal' spectra (average tissue-specific spectra labeled as a single tissue pattern by experts) in the 12 datasets for each tissue pattern are shown in green. Two 'ideal' spectra are highlighted in red and black to illustrate typical variability. (b) Recovered spectra using hierarchical non-negative matrix factorization (hNMF) across the 12 datasets for each tissue pattern are shown in green. The red and black spectra in (b) correspond to the same datasets as in (a). 
itself, without the need for a calibration mechanism (e.g. feature extraction) to bring the datasets to a common ground. Indeed, variations in tissue-specific MRSI spectra from different patients with GBM can be observed between individuals as a result of interpatient anatomical variability and of measurement conditions (in particular, magnetic field inhomogeneities). Thus, the 'ideal' spectra obtained from expert labeling do not reflect the exclusive contribution from a single homogeneous tissue in MRSI data, but should be understood as being representative spectra for the most distinct patterns contained within the considered MRSI dataset. The variations are higher for abnormal tissue, i.e. tumor and necrosis, than for normal tissue (see Fig. 8). Metabolite concentrations also change during the temporal development of tumors, depending on factors such as tissue perfusion, supply of nutrients, cell density, etc. This suggests that the use of common (simulated) tissue-specific spectral profiles to fit data from all patients would result in some inaccuracies.

In this study, the performance evaluation of hNMF was carried out in a controlled environment, i.e. the in vivo data used for method validation came from patients with histopathologically confirmed GBM, chosen to contain necrotic tissue; in addition, expert quality control was performed. However, hNMF should ideally be applied as an initial data exploration tool to differentiate tissue patterns for any brain lesion, i.e. as a compact manner of representing the most important information in MRSI datasets to clinicians. With regard to quality control, recent studies have shown the potential for automation (24); such methods are based on supervised classification and require a training step on a large number of expert-labeled spectra, therefore falling outside the scope of this article. However, in the absence of an automatic preprocessing step for quality control, practical advice for ${ }^{1} \mathrm{H}$ MRSI data acquired under similar conditions to those in this study would be to crop the excitation volume by two to three rows and columns of voxels at each side in order to safely eliminate the most common artifacts: chemical shift displacement effect and lipid contamination.

Furthermore, the number of tissue-specific sources should be detected automatically. The most common choice would be between two or three tissue patterns. When one of the two sources obtained in step 1 presents obvious characteristics of necrosis [i.e. Lips/(Lips + Cho + NAA) $>60 \%$, as in ref. (2)], it is certainly beneficial to continue with step 2 and step 3 of hNMF in order to obtain three sources. If necrosis is not present, the problem is reduced to the application of only one-level NMF with two sources (i.e. step 1 of hNMF).

Currently, our results are significant for patients with GBM, because the differentiation of viable tumor and necrosis is an indicator of the level of aggressiveness, and the estimation of the corresponding location of each tissue pattern might provide additional guidance for surgery or radiotherapy. However, the potential of hNMF is not limited to tissue pattern differentiation of GBMs using MRSI data. In principle, this hierarchical method could be adapted to solve other problems requiring more than two sources or more levels.

\section{Acknowledgements}

This research was supported by Research Council KUL: GOA MaNet, CoE EF/05/006 Optimization in Engineering (OPTEC), PFV/10/002 (OPTEC), IDO 08/013 Autism, several PhD/postdoctoral and fellow grants; Flemish Government: FWO: PhD/postdoctoral grants, projects: G.0427.10N (Integrated EEG-fMRI), G.0108.11 (Compressed Sensing), G.0869.12N (Tumor Imaging), research communities
(ICCoS, ANMMM); IWT: TBM070713-Accelero, TBM070706-IOTA3, TBM080658-MRI (EEG-fMRI), PhD grants; IBBT: Belgian Federal Science Policy Office: IUAP P6/04 (DYSCO, 'Dynamical systems, control and optimization', 2007-2011); ESA AO-PGPF-01, PRODEX (CardioControl) C4000103224; EU: RECAP 209G within INTERREG IVB NWE programme, EU HIP Trial FP7-HEALTH/2007-2013 ( $n^{\circ} 260777$ ); National Natural Science Foundation of China (61271287). Y. Li thanks the China Scholarship Council for financial support.

\section{REFERENCES}

1. Kleihues P, Cavenee WK. Pathology and Genetics of Tumours of the Nervous System. IARC Press: Lyon; 2000.

2. Croitor SA, Martinez-Bisbal MC, Van Huffel S, Cerda JM, Sima DM, Celda B. Ex vivo high resolution magic angle spinning metabolic profiles describe intratumoral histopathological tissue properties in adult human gliomas. Magn. Reson. Med. 2011; 65: 320-328.

3. Law M, Yang S, Wang H, Babb JS, Johnson G, Cha S, Knopp EA, Zagzag D. Glioma grading: sensitivity, specificity, and predictive values of perfusion MR imaging and proton MR spectroscopic imaging compared with conventional MR imaging. Am. J. Neuroradiol. 2003; 24: 1989-1998.

4. Kondziolka D, Lunsford LD, Martinez AJ. Unreliability of contemporary neurodiagnostic imaging in evaluating suspected adult supratentorial (low-grade) astrocytoma. J. Neurosurg. 1993; 74: 536-536.

5. Nelson SJ. Magnetic resonance spectroscopic imaging. IEEE Eng. Med. Biol. 2004; 23: 30-39.

6. Gadian D. NMR and its Applications to Living Systems, 2nd edn. Oxford Science Publishers: Oxford, 1995.

7. Rutter A, Hugenholtz H, Saunders JK, Smith ICP. Classification of brain tumors by ex vivo ${ }^{1} \mathrm{H}$ NMR spectroscopy. J. Neurochem. 1995; 64: 1655-1661.

8. Howe FA, Barton SJ, Cudlip SA, Stubbs M, Saunders DE, Murphy JR, Opstad KS, Doyle VL, McLean MA, Bell BA, Griffiths JR. Metabolic profiles of human brain tumours using quantitative in vivo ${ }^{1} \mathrm{H}$ magnetic resonance spectroscopy. Magn. Reson. Med. 2003; 49: 223-232.

9. Delikatny EJ, Chawla S, Leung D-J, Poptani H. MR-visible lipids and the tumor microenvironment. NMR Biomed. 2011; 24: 592-611.

10. Luts J, Laudadio T, Idema AJ, Simonetti AW, Heerschap A, Vandermeulen D, Suykens JAK, Van Huffel S. Nosologic imaging of the brain: segmentation and classification using MRI and MRSI. NMR Biomed. 2009; 22: 374-390.

11. Wright AJ, Fellows G, Byrnes TJ, Opstad KS, Mclntyre DJO, Griffiths JR, Bell BA, Clark CA, Barrick TR, Howe FA. Pattern recognition of MRSI data shows regions of glioma growth that agree with DTI markers of brain tumor infiltration. Magn. Reson. Med. 2009; 62: 1646-1651.

12. Ochs MF, Stuyanova RS, Arias-Mendoza F, Brown TR. A new method for spectral decomposition using a bilinear Bayesian approach. J. Magn. Reson. 1999; 137: 161-176.

13. Szabo de Edelenyi F, Simonetti AW, Postma G, Huo R, Buydens LMC. Application of independent component analysis to ${ }^{1} \mathrm{H}$ MR spectroscopic imaging exams of brain tumours. Anal. Chim. Acta 2005; 544: 36-46.

14. Lee DD, Seung HS. Learning the parts of objects by non-negative matrix factorization. Nature, 1999; 401: 788-791.

15. Sajda P, Du S, Brown TR, Stoyanova R, Shungu DC, Mao X, Parra LC. Nonnegative matrix factorization for rapid recovery of constituent spectra in magnetic resonance chemical shift imaging of the brain. IEEE Trans. Med. Imaging, 2004; 23: 1453-1465.

16. Su Y, Thakur SB, Karimi S, Du S, Sajda P, Huang W, Parra LC. Spectrum separation resolves partial-volume effect of MRSI as demonstrated on brain tumor scans. NMR Biomed. 2008; 21: 1030-1042.

17. Du S, Mao X, Sajda P, Shungu DC. Automated tissue segmentation and blind recovery of ${ }^{1} \mathrm{H}$ MRS imaging spectral patterns of normal and diseased human brain. NMR Biomed. 2008; 21: 33-41.

18. Lawson CL, Hanson RJ. Solving Least-Squares Problems, Prentice-Hall, Chapter 23, 1974; 161.

19. Li Y, Sima DM, Van Cauter S, Himmelreich U, Pi Y, Van Huffel S. Simulation study of tissue type differentiation using non-negative matrix factorization. Proceedings of BIOSIGNALS 2012 International Conference on Bioinspired Systems and Signal Processing, Vilamoura, Algarve, Portugal, February, 2012; 212-217. 
20. Li Y, Sima DM, Van Cauter S, Himmelreich U, Pi Y, Van Huffel S. Tissue type differentiation for brain glioma using non-negative matrix factorization. Proceedings of BIOSIGNALS 2012 International Conference on Bioinspired Systems and Signal Processing, Vilamoura, Algarve, Portugal, February, 2012; 25-31.

21. Bottomley PA. Spatial localization in NMR-spectroscopy in vivo. Ann. N. Y. Acad. Sci. 1987; 508: 333-348.

22. Louis DN, Ohgaki H, Wiestler OD, Cavenee WK, Burger PC, Jouvet A, Scheithauer BW, Kleihues P. The 2007 WHO classification of tumours of the central nervous system. Acta Neuropathol. 2007; 114: 97-109.

23. Kreis R. Issues of spectral quality in clinical ${ }^{1} \mathrm{H}$-magnetic resonance spectroscopy and a gallery of artifacts. NMR Biomed. 2004; 17: 361-381.

24. Wright AJ, Arús C, Wijnen JP, Moreno-Torres A, Griffiths JR, Celda B, Howe FA. Automated quality control protocol for MR spectra of brain tumors. Magn. Reson. Med. 2008; 59: 1274-1281.

25. Laudadio T, Mastronardi N, Vanhamme L, Van Hecke P, Van Huffel S. Improved Lanczos algorithms for blackbox MRS data quantitation. J. Magn. Reson. 2002; 157: 292-297.

26. Poullet JB. Quantification and classification of magnetic resonance spectroscopic data for brain tumor diagnosis. PhD Thesis, Leuven; 2008. Available at: http://homes.esat.kuleuven.be/ biomed/software. php\#SpidGUI/ [accessed 01 January 2012].

27. Lin CJ. Projected gradient methods for nonnegative matrix factorization. Neural Comput. 2007; 19: 2756-2779.

28. Berry M, Browne M, Langville A, Pauca V, Plemmons R. Algorithms and applications for approximate non-negative matrix factorization. Comput. Stat. Data. Anal. 2007; 52: 155-173.

29. Cichocki A, Zdunek R, Amari S. Hierarchical ALS algorithms for nonnegative matrix and $3 \mathrm{D}$ tensor factorization. Lect. Notes. Comput. Sci. 2007; 4666: 169-176.

30. Cichocki A, Phan AH. Fast local algorithms for large scale nonegative matrix and tensor factorizations. IEICE Trans. Fund. Electron. Comm. Comput. Sci. 2009; 3: 708-721.

31. Gillis N. Nonnegative matrix factorization complexity, algorithms and applications. PhD Thesis, Louvan-La-Neuve; 2011.
32. Duyn JH, Gillen J, Sobering G, van Zijl PC, Moonen CT. Multisection proton MR spectroscopic imaging of the brain. Radiology 1993; 188: 277-282.

33. Majós $C$, Julià-Sapé $M$, Alonso J, Serrallonga M, Auilera $C$, Acebes JJ, Arús C, Gili J. Brain tumor classification by proton MR spectroscopy: comparison of diagnostic accuracy at short and long TE. Am. J. Neuroradiol. 2004; 25: 1696-1704.

34. Ortega-Martorell S, Lisboa PJG, Vellido A, Julià-Sapé M, Arús C. Nonnegative matrix factorisation methods for the spectral decomposition of MRS data from human brain tumours. BMC Bioinformatics 2012; 13: 38.

35. Ladroue C, Howe FA, Griffiths JR, Tate AR. Independent component analysis for automated decomposition of in vivo magnetic resonance spectra. Magn. Reson. Med. 2003; 50: 697-703.

\section{APPENDIX A: PARAMETERS FOR SIMULATED MRSI SIGNALS}

We simulated time-domain MRSI signals as a combination of nine metabolite signals based on the formula:

$$
\begin{aligned}
y(t)=\sum_{k=1}^{9} \operatorname{Amp}_{k} & \times \operatorname{Met}_{k}(t) \times \exp \left(j \times \text { phase }_{k}-\text { damp }_{k}\right. \\
& \left.\times t+j \times \operatorname{freq}_{k} \times t\right)+ \text { noise }
\end{aligned}
$$

where $\mathrm{Amp}_{k}$ is the amplitude of metabolite $k$, damp $\mathrm{p}_{k}$ is the damping of metabolite $k$, freq f $_{k}$ is the frequency shift of metabolite $k$, phase $e_{k}$ is the phase shift of metabolite $k$, Met ${ }_{k}$ is the time-domain metabolite signal for metabolite $k$ and $j=\operatorname{sqrt}(-1)$.

The values in Tables A1-A3 are adapted from the spectral simulation module of SPID software (26). Metabolites measured in vitro are normalized to the same unit. The simulated lipids are normalized to the same scale as the other metabolites, i.e. norm(Lip1) = norm(NAA) and norm(Lip2) $=$ norm(NAA).

Table A1. Normal

\begin{tabular}{llcccccccccc} 
& \multicolumn{1}{c}{ Metabolite } \\
\cline { 2 - 10 } & & Cre & Glu & Myo & PCh & NAA & Ala & Lac & Lip1 & Lip2 \\
\hline \multirow{2}{*}{ Amplitude } & Mean $\left(10^{3}\right)$ & 389 & 444 & 307 & 123 & 552 & 96 & 12 & 1.3 & 1.5 \\
& SD $\left(10^{3}\right)$ & 76 & 96 & 93 & 6 & 93 & 60 & 8.9 & 0.8 & 0.7 \\
Damping & Mean $\left(10^{-3}\right)$ & 9.4 & 9.0 & 8.5 & 6.6 & 8.4 & 6.9 & 6.2 & 4.1 & 8.7 \\
& SD $\left(10^{-3}\right)$ & 1.4 & 2.3 & 2.5 & 3.6 & 2.3 & 4.4 & 4.2 & 4.4 & 3.1 \\
Frequency shift & & 0 & 0 & 0 & 0 & 0 & 0 & 0 & 0 & 0 \\
Phase shift & & 0 & 0 & 0 & 0 & 0 & 0 & 0 & 0
\end{tabular}

Ala, alanine; Cre, creatine; Glu, glutamate; Lac, lactate; Lip1, lipid at 1.3 ppm; Lip2, lipid at 0.9 ppm; Myo, myo-inositol; NAA, $\mathrm{N}$-acetylaspartate; PCh, phosphocholine; SD, standard deviation.

Table A2. Tumor

\begin{tabular}{|c|c|c|c|c|c|c|c|c|c|c|}
\hline & & \multicolumn{9}{|c|}{ Metabolite } \\
\hline & & Cre & Glu & Myo & PCh & NAA & Ala & Lac & Lip1 & Lip2 \\
\hline \multirow[t]{2}{*}{ Amplitude } & Mean $\left(10^{3}\right)$ & 304 & 374 & 306 & 173 & 202 & 295 & 317 & 1.7 & 1.8 \\
\hline & $\operatorname{SD}\left(10^{3}\right)$ & 146 & 182 & 149 & 108 & 95 & 309 & 328 & 1.0 & 1.0 \\
\hline \multirow[t]{2}{*}{ Damping } & Mean $\left(10^{-3}\right)$ & 9.4 & 9.0 & 8.5 & 6.6 & 8.3 & 6.9 & 6.2 & 4.1 & 8.7 \\
\hline & $\operatorname{SD}\left(10^{-3}\right)$ & 1.4 & 2.3 & 2.5 & 3.6 & 2.3 & 4.4 & 4.2 & 4.4 & 3.1 \\
\hline \multicolumn{2}{|c|}{ Frequency shift } & 0 & 0 & 0 & 0 & 0 & 0 & 0 & 0 & 0 \\
\hline \multicolumn{2}{|c|}{ Phase shift } & 0 & 0 & 0 & 0 & 0 & 0 & 0 & 0 & 0 \\
\hline
\end{tabular}

Ala, alanine; Cre, creatine; Glu, glutamate; Lac, lactate; Lip1, lipid at 1.3 ppm; Lip2, lipid at 0.9 ppm; Myo, myo-inositol; NAA, $\mathrm{N}$-acetylaspartate; PCh, phosphocholine; SD, standard deviation. 
Table A3. Necrosis

\begin{tabular}{|llcccccccccc} 
& \multicolumn{1}{c}{} & \multicolumn{1}{c}{ Metabolite } \\
\cline { 2 - 10 } & & Cre & Glu & Myo & PCh & NAA & Ala & Lac & Lip1 & Lip2 \\
\hline Amplitude & Mean $\left(10^{3}\right)$ & 10 & 74 & 44 & 6.5 & 4.9 & 97 & 43 & 8864 & 3244 \\
& SD $\left(10^{3}\right)$ & 107 & 134 & 9.4 & 30 & 70 & 60 & 33 & 3.7 & 2.4 \\
Damping & Mean $\left(10^{-3}\right)$ & 7.8 & 9.3 & 7.4 & 8.3 & 9.8 & 9.5 & 10 & 89 & 70 \\
& SD $\left(10^{-3}\right)$ & 3.9 & 1.9 & 3.8 & 3.3 & 1.2 & 2.1 & 0.4 & 0.3 & 0.1 \\
Frequency shift & & 0 & 0 & 0 & 0 & 0 & 0 & 0 & 0 & 0 \\
Phase shift & & 0 & 0 & 0 & 0 & 0 & 0 & 0 & 0
\end{tabular}

Ala, alanine; Cre, creatine; Glu, glutamate; Lac, lactate; Lip1, lipid at 1.3 ppm; Lip2, lipid at 0.9 ppm; Myo, myo-inositol; NAA, $\mathrm{N}$-acetylaspartate; $\mathrm{PCh}$, phosphocholine; SD, standard deviation. 